\title{
Stochastic modelling and feedback control of bistability in a turbulent bluff body wake
}

\author{
R. D. Brackston ${ }^{1, \dagger}$, J. M. García de la Cruz $^{1}$, A. $\mathbf{W y n n}^{1}$, G. $\operatorname{Rigas}^{1, \dagger}$ and \\ J. F. Morrison ${ }^{1}$ \\ ${ }^{1}$ Department of Aeronautics, Imperial College London, London SW7 2AZ, UK
}

(Received 7 March 2016; revised 26 June 2016; accepted 20 July 2016; first published online 10 August 2016)

A specific feature of three-dimensional bluff body wakes, flow bistability, is a subject of particular recent interest. This feature consists of a random flipping of the wake between two asymmetric configurations and is believed to contribute to the pressure drag of many bluff bodies. In this study we apply the modelling approach recently suggested for axisymmetric bodies by Rigas et al. (J. Fluid Mech., vol. 778, 2015, R2) to the reflectional symmetry-breaking modes of a rectilinear bluff body wake. We demonstrate the validity of the model and its Reynolds number independence through time-resolved base pressure measurements of the natural wake. Further, oscillating flaps are used to investigate the dynamics and time scales of the instability associated with the flipping process, demonstrating that they are largely independent of Reynolds number. The modelling approach is then used to design a feedback controller that uses the flaps to suppress the symmetry-breaking modes. The controller is successful, leading to a suppression of the bistability of the wake, with concomitant reductions in both lateral and streamwise forces. Importantly, the controller is found to be efficient, the actuator requiring only $24 \%$ of the aerodynamic power saving. The controller therefore provides a key demonstration of efficient feedback control used to reduce the drag of a high-Reynolds-number three-dimensional bluff body. Furthermore, the results suggest that suppression of large-scale structures is a fundamentally efficient approach for bluff body drag reduction.

Key words: drag reduction, flow control, low-dimensional models

\section{Introduction}

For many three-dimensional wakes, a prominent feature that is believed to contribute to pressure drag is the so-called bistability. This feature consists of an instantaneous asymmetry of the wake, even under nominally symmetric flow conditions, and a random switching between two such asymmetric states. Bistability was first recognised by Herry et al. (2011), who observed mean flow asymmetry and high sensitivity to side slip angle for the flow over a three-dimensional double backward-facing step.

$†$ Email address for correspondence: r.brackston13@imperial.ac.uk

$\$$ Present address: California Institute of Technology, Pasadena, California 91125, USA. 

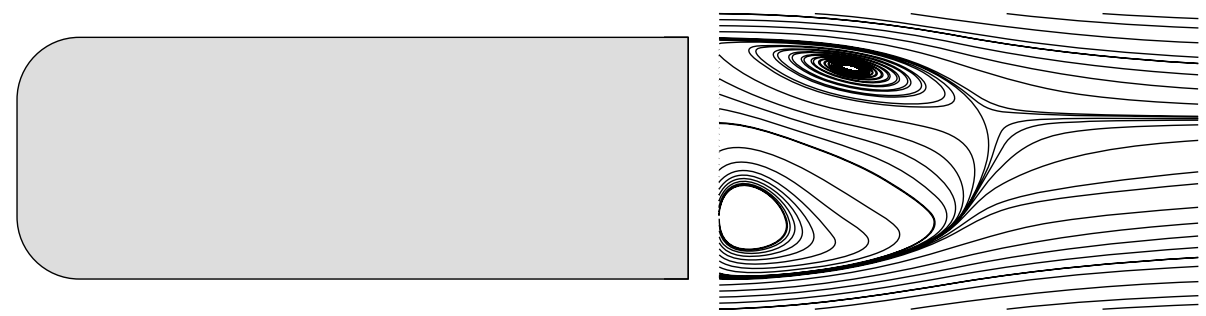

FIGURE 1. Plan view of the Ahmed body showing a conditional average of one of the symmetry-breaking states of the wake, obtained from particle image velocimetry. The streamlines demonstrate the asymmetry, showing the stationary vortex adjacent to the base.

(a)

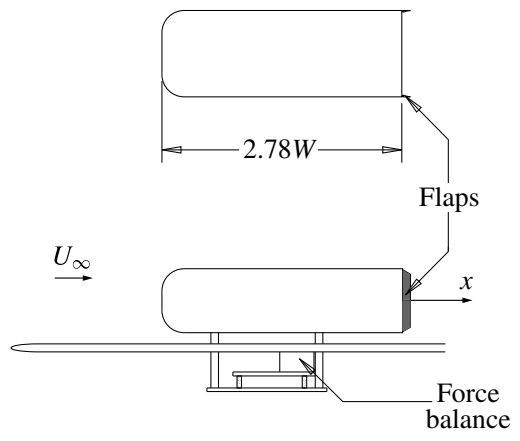

(b)

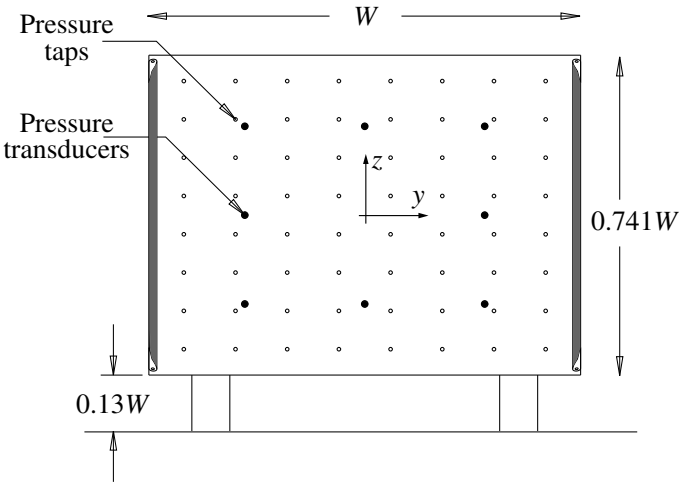

FIGURE 2. View of the Ahmed body showing the overall assembly (a) and detailed view of the base $(b)$. The model width $W$ is $0.216 \mathrm{~m}$. The positioning of the flaps, pressure sensors and force balance is shown.

Since then, a number of studies have examined this same feature on the body of Ahmed, Ramm \& Faltin (1984). This so-called Ahmed body, shown in figures 1 and 2 , provides a generic representation of a road vehicle, including the essential features of a real vehicle flow field such as three-dimensional separation and ground effect.

At low Reynolds numbers (Re), of order 100, the flow behind the Ahmed body is steady and is characterised by reflectional symmetry with respect to the vertical symmetry plane of the body (Grandemange, Cadot \& Gohlke 2012). Due to the presence of the ground, a top-bottom asymmetry exists with respect to the horizontal symmetry plane. Increasing the Reynolds number, the flow undergoes a steady supercritical bifurcation and the resulting steady flow loses the remaining spatial symmetry. The flow breaks the left-right reflectional symmetry and, depending on the initial conditions, relaxes in one of two possible stable asymmetric states. For even higher Reynolds numbers, the flow becomes unsteady and periodic shedding starts to occur. These two regimes were observed for $R e>340$ and $R e>410$, respectively (Grandemange et al. 2012).

It has been recently shown that the two reflectional symmetry-broken (RSB) states observed in the laminar regime persist at much higher Reynolds numbers $\left(\operatorname{Re} \approx 10^{5}\right)$ (Grandemange, Gohlke \& Cadot 2013c; Cadot, Evrard \& Pastur 2015; Volpe, Devinant $\&$ Kourta 2015) and for a number of different geometric configurations (Grandemange, 
Gohlke \& Cadot 2013b). For a body aligned with the flow, the asymmetric structure is demonstrated to flip randomly between two asymmetric states, each the mirror image of the other. In the long-time average this 'flipping' leads to a statistically symmetric wake, while instantaneously the wake is typically asymmetric with an associated lateral force on the body. The structure of this asymmetric flow is displayed in figure 1, and consists of a static vortex adjacent to the base towards one side, leading to a low-pressure region. For a more detailed description the reader is referred to Evrard et al. (2015, figure 11). The flipping process has two time scales associated with it, the flipping period $(T)$ defining the average time between flips, and the instability time scale $(\delta)$ defining the duration of a flipping event. The former is generally many orders of magnitude greater than the latter. Previous studies have examined the statistics of the flipping process, including the flipping period, with respect to ground clearance (Cadot et al. 2015) and flow alignment (Volpe et al. 2015), but none has looked in detail at the flipping dynamics. The instability time scale $\delta$ therefore remains to be investigated in detail.

The RSB modes observed for high-Reynolds-number rectilinear three-dimensional (3-D) bluff body wakes are qualitatively similar to the asymmetry seen in the wakes of other 3-D bluff bodies, most notably in axisymmetric cases. For example, Rigas et al. (2014) and Grandemange, Gohlke \& Cadot (2014a) both demonstrate that, instantaneously, the wakes of respectively a bullet-shaped body and a sphere are asymmetric but that this asymmetry is not present over long-time averages. For such axisymmetric wakes this feature is again linked to the spatial symmetry breaking seen at low Reynolds numbers (Bury \& Jardin 2012), shown to result from a supercritical pitchfork bifurcation (Fabre, Auguste \& Magnaudet 2008; Meliga, Chomaz \& Sipp 2009). For the bullet-shaped body, this link with the low-Reynolds-number bifurcations has allowed an accurate model to be developed for the turbulent dynamics (Rigas et al. 2015). Here we apply the same approach to the wake of a rectilinear body, obtaining a model that accurately captures the dynamics of the RSB modes including the time scale of the instability.

A number of studies of both axisymmetric and rectilinear bodies have looked at methods to control the wake, including control of the spatial symmetry-breaking mode. For example, Grandemange, Gohlke \& Cadot (2014b) and Cadot et al. (2015) both applied a vertical control cylinder in the wake of the Ahmed body, finding that if correctly located, the bistable behaviour could be suppressed, achieving a small concomitant drag decrease. More recently, Evrard et al. (2015) found that a base cavity was also able to achieve bistability suppression with even larger drag reductions. For the axisymmetric body, similar results have been achieved using a passive slit around the perimeter of the base (García de la Cruz, Oxlade \& Morrison 2016). While these passive methods have proven effective, the requirements for suppression of the spatial symmetry-breaking mode remain unclear. Moreover, it remains to be seen whether the mode can be suppressed without significant geometric modification. This motivates the use of active feedback control techniques.

Closed-loop control of fluid flows such as wakes has historically proven to be challenging because of infinite degrees of freedom of the fluid continuum and strongly nonlinear dynamics. While some flow control methods such as extremum seeking (Henning et al. 2008) and machine learning control (Gautier et al. 2015) are model free, many more rely on having a mathematical model, and often a linear model, of the fluid system. For example, black-box linear models have often been used for convectively unstable flows such as the backward-facing step (Barbagallo et al. 2012; Dahan, Morgans \& Lardeau 2012; Gautier \& Aider 2014), because such 
flows behave like a linear amplifier, thus allowing linear models to be obtained for various input-output behaviours. For absolutely unstable flows that operate on a limit cycle it is much more challenging, as the flow is already operating in a fully nonlinear regime. In such cases, a linear fit may be obtained about an operating point, but not about the desired operating condition. For numerical simulations, the unstable system may still be identified since the base flow can be evaluated directly along with the perturbation dynamics (Barbagallo, Sipp \& Schmid 2009). For experimental flows this is not possible. Another solution has been to obtain a working controller by trial and error, and then to examine the dynamics of the stabilised system (Morgans \& Dowling 2007; Illingworth, Morgans \& Rowley 2012). While this is an attractive solution in some cases, empirical design of an effective controller for a turbulent high-Re flow is usually not possible. Therefore, having a priori the form of an accurate model of the flow is of great benefit for feedback control. Moreover, we may expect to be able to achieve better control through knowledge of the flow physics than through the use of generic methods (Ho \& Pepyne 2002). The nonlinear Langevin model that we develop here for the RSB modes provides just such a model that enables informed design of a feedback controller.

In this paper we use oscillating flaps along with pressure measurements to provide further insight into the dynamics of the bistability, specifically estimating the time scale of the instability and the variation of the dynamics with $R e$. The dynamics is then linked to the modelling approach of Rigas et al. (2015), wherein the large-scale flow structure of the bistability is modelled by a simple nonlinear Langevin equation, the stochastic term modelling the effect of the turbulence. In contrast to many previous flow control strategies, the present modelling approach allows us to linearise analytically a low-dimensional model, thereby enabling us to design a feedback controller based on the equations governing the laminar large-scale structures. This controller is designed with the aim of suppressing the asymmetric large-scale structure of the RSB mode, and is demonstrated to reduce both the pressure drag and the lateral force on the body.

The paper is outlined as follows. The experimental set-up is detailed in $\S 2$, before the modelling and open-loop results are described in $\S 3$. The design and results of the feedback controller are then given in $\S 4$, before conclusions are made in $\S 5$.

\section{Experimental set-up}

The experimental set-up is shown in figure 2. Experiments were carried out in a closed-circuit wind tunnel with a test section measuring $1.37 \mathrm{~m} \times 1.22 \mathrm{~m} \times 3.00 \mathrm{~m}$. The model blockage ratio was $2.1 \%$ and the free-stream turbulence intensity was less than $0.1 \%$. The tunnel is controlled using a proportional integral derivative (PID) controller to achieve the desired free-stream velocity to an accuracy of $0.25 \%$.

The experimental model consists of a scaled-down, flat-backed Ahmed body of the proportions specified by Ahmed et al. (1984). The base of the model measures $0.216 \mathrm{~m} \times 0.160 \mathrm{~m}$, giving $R e$ of $O\left(10^{5}\right)$. The model sits $0.028 \mathrm{~m}$ above a raised floor, ensuring minimal boundary layer thickness over the tunnel floor while maintaining the correct ground effect. A force balance, situated out of the air flow, provides the connection between the model and the tunnel, enabling accurate measurement of the total force and moment acting on the body.

In addition to the force balance, the model is instrumented with eight Endevco 8507C pressure transducers for fluctuating pressure measurements and an ESP-DTC pressure scanner supplying 64 static pressure measurements distributed over the 
model base. The static pressure measurements are used to calculate the timeaveraged statistics while the pressure transducers are used for time-resolved pressure measurements and feedback control.

Forcing of the wake is achieved using two flaps located at the trailing edges of the sides of the body, each $0.019 \mathrm{~m}$ in streamwise length and running the height of the body. The flaps are mounted on hinges driven by internal motors powered through an amplifier. The flaps are given a natural equilibrium and oscillatory dynamics through the action of internal springs. The angle of each flap is measured using a 12-bit magnetic encoder while the power supplied to the flaps is monitored through measurement of the supply voltage and the current through the motors.

All channels are sampled simultaneously at $25 \mathrm{kHz}$ with the exception of the pressure scanners that are sampled at $250 \mathrm{~Hz}$, and synchronised with every hundredth analogue sample. For open-loop forcing of the wake, the mean angles, fluctuation amplitudes and phases of the flaps are PID-controlled to ensure uniform forcing at all frequencies. Feedback control was achieved using a National Instruments Real-Time PXI running a discrete time controller at $5 \mathrm{kHz}$. The controller obtains pressure measurements from six of the Endevco transducers and outputs two voltages that are sent to the motors.

\section{Modelling and open-loop investigations}

In this section we provide the methodology for deriving a physical model capturing the dynamic evolution of the RSB mode, and identify its unknown parameters experimentally. The basis for the model is the observation that the bistability of the turbulent flow results from a persistence of the laminar global modes at high Reynolds numbers. The effect of turbulence on the deterministic nonlinear dynamics governing the RSB mode is modelled as stochastic forcing, as in Rigas et al. (2015). The model is also extended to capture the response of the mode to open-loop forcing provided by the flaps, in anticipation of its use for feedback control design.

For rectilinear bodies it has been shown that the spatial symmetry-breaking mode occurs in only one of the two dimensions, the specific dimension depending upon the aspect ratio and ground effect (Grandemange et al. 2013b). For the Ahmed body wake this feature occurs only in the lateral dimension (see figure 2); we therefore require some metric that captures this feature from our experimental data. While there are many choices for this metric, here, as in Rigas et al. (2014) and Volpe et al. (2015), we take the lateral component of the centre of pressure (CoP) location. Nondimensionalised by the body width, this is defined as

$$
r(t)=\frac{1}{W \iint_{A} p(y, t) \mathrm{d} A} \iint_{A} p(y, t) y \mathrm{~d} A,
$$

where $p(y, t)$ is the local pressure obtained from either the static taps or the pressure transducers, $y$ is the lateral coordinate and $A$ is the area over the base of the body. Henceforth we will use the parameter $r$ to describe the symmetry-breaking mode of the wake and, when displaying experimental results, use the metric defined by (3.1).

\subsection{Modelling}

Our modelling of the RSB mode of the wake follows the modelling approach of Rigas et al. (2015). To begin, the normal form of the spatial symmetry-breaking mode of the 

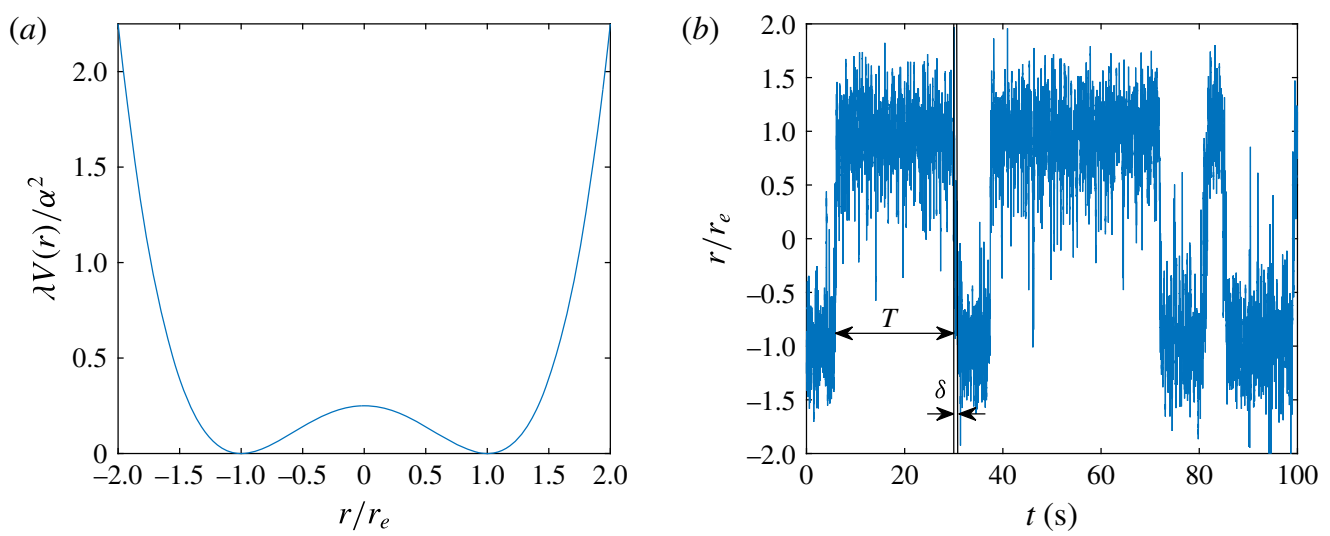

FIgURE 3. (Colour online) (a) The potential well $V(r)$ for $\theta=0$, and $(b)$ an example time series demonstrating the time scales $T$ and $\delta$.

wake can be written as

$$
\dot{\boldsymbol{x}}=\alpha \boldsymbol{x}-\lambda \boldsymbol{x}^{3} .
$$

This equation describes a supercritical pitchfork bifurcation where $\boldsymbol{x}$ is the bifurcated mode, $\dot{\boldsymbol{x}}$ the time derivative, $\alpha$ the growth rate and $\lambda$ the saturation parameter.

In the turbulent regime and for the rectilinear bluff body wake of our experiment, (3.2) can now be written in terms of the mode $r$ as

$$
\dot{r}=\alpha r-\lambda r^{3}+b \theta_{t-\tau}+\sigma \xi(t) .
$$

Here, the first additional term $b \theta_{t-\tau}$ models the effect of the forcing by the flaps. The flaps may be expected to deflect the shear layers by an amount determined by their angle $\theta$. They thereby provide a lateral momentum flux that forces the mode $r$, according to some linear scaling factor $b$. Given that any shear layer perturbation must be advected, it is natural to also incorporate an advective time delay $\tau$. We will demonstrate later that the nature of this term fits well with the experimental observations ( $\S \S 3.3$ and 3.4). The additional term $\sigma \xi(t)$ is used to model the broadband forcing due to turbulence acting on the large-scale structure of the mode, where $\xi$ is a normally distributed random variable and $\sigma^{2}$ gives the variance (noise intensity). This is intended to capture all of the remaining perturbations in the flow that will cause the mode $r$ to change. Given the coherence of the mode described by $r$, we may expect the term $\sigma \xi(t)$ to be relatively small. Furthermore, for the flaps to have good authority over the flow, we may require the term $b \theta_{t-\tau}$ to be relatively large. We will see in $\$ 3.5$ that this is indeed the case.

The system described by (3.3) is one of a state $r$ moving in a double-well potential $V(r)$, as illustrated in figure $3(a)$. The turbulent forcing perturbs the state within this well, while the parameter $\theta$ is able to skew the well in either direction. Such systems are commonplace and have been studied extensively in the past (see for example Gammaitoni et al. 1998), therefore we will summarise only the key features here. In the absence of noise and for $\theta=0$, the system has three points of equilibrium: one unstable at $r=0$ and two stable at $r= \pm \sqrt{\alpha / \lambda}= \pm r_{e}$. The state $r$ will therefore tend to move in a region around one of the two stable equilibrium points until sufficiently 
perturbed by the noise to jump to the other. The mean time between such jumps, $T$, is illustrated in figure $3(b)$, and may be shown to be given by (Zwanzig 2001)

$$
T=\frac{\pi}{2 \alpha} \exp \left(\frac{\alpha r_{e}^{2}}{2 \sigma^{2}}\right) .
$$

The second time scale of the process is the instability time scale associated with the duration of a flipping event, as demonstrated in figure $3(b)$, and is approximately given by $\delta \approx 1 / \alpha$. The ratio of these two time scales therefore depends upon the ratio $\sigma^{2} / \alpha$ and determines the proportion of time spent at or near the equilibrium positions.

In the long-time average the distribution of values for $r$ may be given analytically by the steady state Fokker-Planck equation (Risken 1996). The stationary probability density function (PDF) for the system described by (3.3) with constant $\theta$ is

$$
\begin{aligned}
P(r) & =C \exp \left(-\frac{2 V(r)}{\sigma^{2}}\right) \\
& =C \exp \left(\frac{\alpha}{\sigma^{2}}\left(r^{2}-\frac{1}{2} \frac{\lambda}{\alpha} r^{4}\right)+\frac{2 b \theta}{\sigma^{2}} r\right),
\end{aligned}
$$

where $C$ is a normalisation constant. This will later allow us to validate the model and aid in establishing the parameters.

The model is also consistent with expectations for the power spectral density (PSD) of the variable $r$. At high frequencies, (3.3) describes diffusive motion since $\dot{r} \approx \sigma \xi$. At very low frequencies, i.e. long time scales, (3.3) leads to a random switching process as observed in practice. For both diffusive motion and low-frequency flipping (Grandemange 2013, § D.2.) the PSD is expected to follow $S_{r r}(\omega) \propto \omega^{-2}$, i.e. the PSD will have a slope of -2 on a $\log -\log$ axis.

\subsection{Unforced flow}

The unforced flow was examined at three different free-stream velocities corresponding to $R e=2.3 \times 10^{5}, 3.0 \times 10^{5}$ and $4.4 \times 10^{5}$. Figure 4(a) shows the PDF for $r$, along with the model prediction from (3.5), using the parameters shown in $\S 3.5$. The PDF demonstrates the asymmetry of the flow since the mode is non-zero, i.e. the PDF peaks at $|r|=r_{e}$, the equilibrium value of (3.3). The results demonstrate both collapse of the data with $R e$ and agreement with the model prediction.

As indicated in (3.5), the unforced $(\theta=0) \mathrm{PDF}$ can be written as a function of two non-dimensional parameters: $\sigma^{2} / \alpha$ and $\lambda / \alpha$. These two parameters determine respectively the sharpness and location of the peak in the PDF and are seen to be largely $R e$ independent. Physically, the parameter $\sigma^{2} / \alpha$ gives the intensity of the noise relative to the growth rate of the instability. The results therefore suggest that as $R e$ changes, any change in growth rate is accompanied by a roughly equal change in the noise intensity. As noted above, this parameter is closely linked to the ratio of the two flipping time scales: a large $\sigma^{2} / \alpha$ corresponds to a larger $\delta / T$. The second parameter, $\lambda / \alpha$, describes the level of asymmetry, and hence the topology of the flow, at the two stable equilibrium points. The results therefore suggest that this topology remains approximately constant.

Figure 4(b) shows the power spectral density of $r$ as a function of the Strouhal number, $S t_{W}=f W / U_{\infty}$. At lower frequencies, a region of $\omega^{-2}$ roll-off is observed for all three $R e$, consistent with the expectations for a randomly bistable process. 

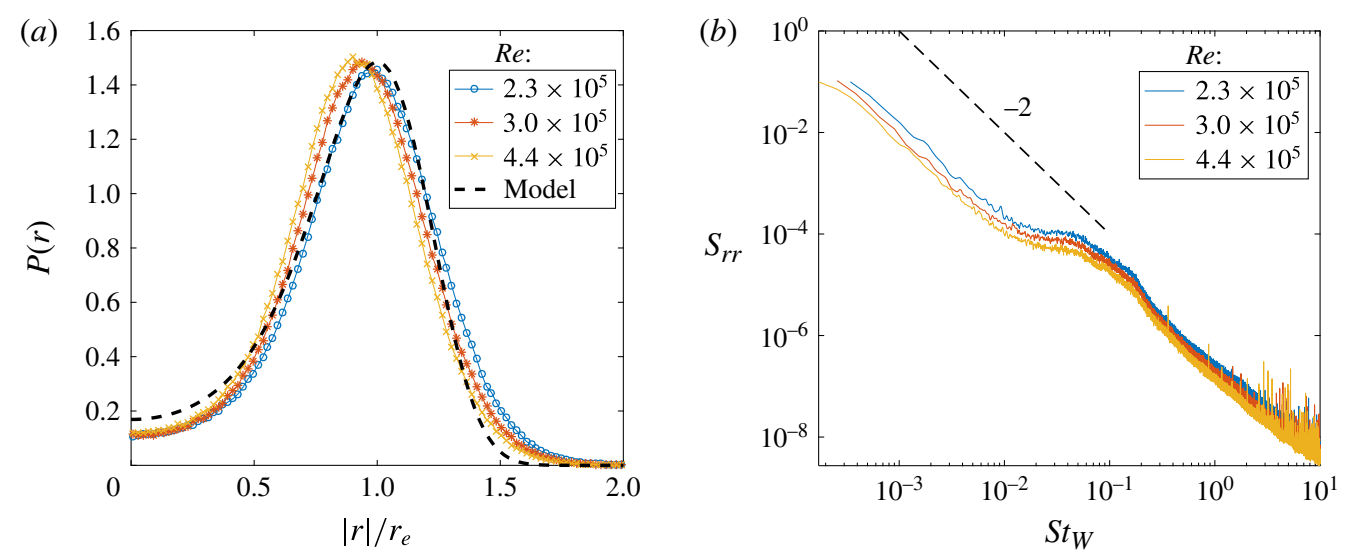

FIGURE 4. (Colour online) Properties of the mode $r$ at the three tested $R e$ : $(a)$ probability density function and $(b)$ power spectral density.

\subsection{Response to harmonic forcing}

While the unforced results of $\S 3.2$ allow assessment of the time-averaged behaviour, full quantification of the process requires knowledge of the time scales quantified by $\alpha$ and $\tau$. These time scales can most accurately be assessed by forcing the flow with the lateral flaps in order to induce repeated flipping of the asymmetric structure. By performing such harmonic forcing at a range of frequencies we obtain a frequency response for the flow and also understand how the flaps affect the drag of the body.

The frequency response is found using a standard system identification technique. The Fourier coefficients corresponding to the frequency of the harmonic forcing are evaluated for both the input and the output. From these coefficients, the gain and phase are evaluated. The input is taken as the mean of the two flap angles, with the sign convention that a positive angle for both flaps corresponds to antisymmetric forcing, i.e. forcing in which when one flap moves inwards the other moves outwards. The output is the metric $r$, approximated using the pressure transducers. The complex gain $G\left(S t_{W}\right)$ is therefore defined as $R\left(S t_{W}\right) / \Theta\left(S t_{W}\right)$ where $R$ and $\Theta$ are respectively the Fourier transforms of $r$ and $\theta$.

Figure 5(a) shows the antisymmetric frequency response of the flow at the three different $R e$, demonstrating that the responses collapse in both magnitude and phase. This shows that the dynamics and flow topology associated with the process remain essentially identical, such that in a dimensionless form the parameters of (3.3) remain constant.

The amplitude of the frequency response shown in the upper part of figure 5(a) can be considered to consist of a number of discrete sections. Typical phase-averaged responses for each of these sections are shown in figure 5(b). At very low frequencies, $S t_{W} \lesssim 10^{-2}$, the response is essentially constant. In this range the flaps simply induce flipping of the asymmetric structure between the two equilibrium points of $\pm r_{e}$. This gives an approximately square-wave output at the forcing frequency, as shown in figure $5(b)$. For $10^{-2}<S t_{W}<8 \times 10^{-2}$ the amplitude response begins to decay as an increasing portion of the forcing period is spent moving between the two extremes of the motion. At $S t_{W} \approx 8 \times 10^{-2}$ the rate of decay rapidly changes, and by a frequency of $S t_{W} \approx 10^{-1}$ the response has decayed by one and a half orders of magnitude. 

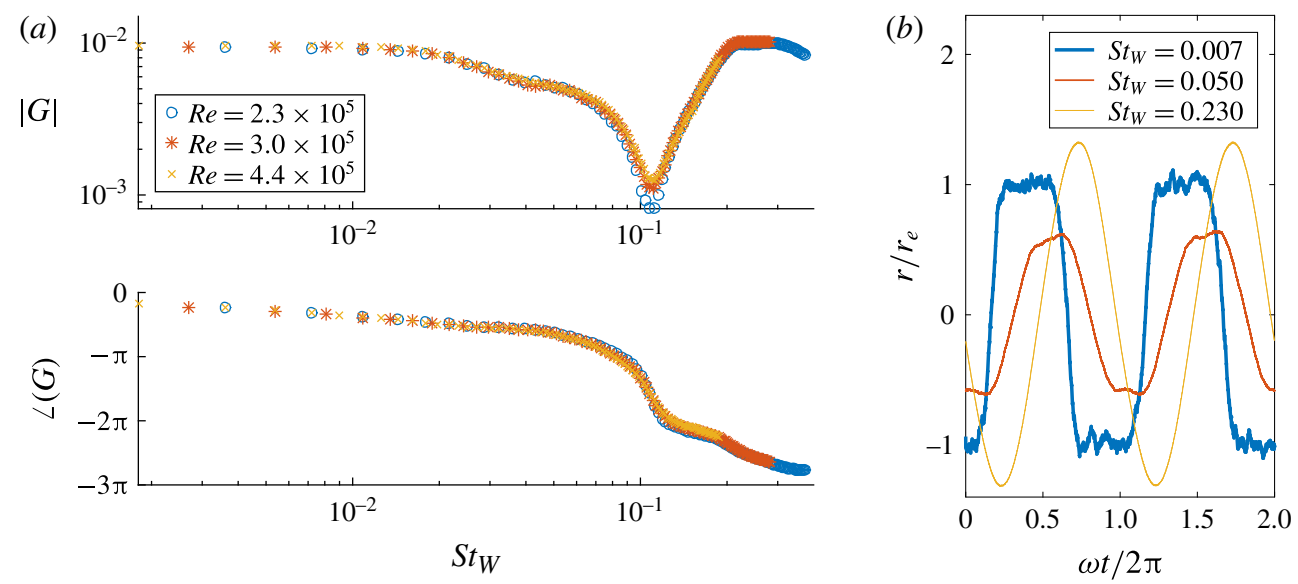

FIgURE 5. (Colour online) (a) Frequency response between the antisymmetric flap oscillation and the mode $r$ at the three $R e$ and $(b)$ phase-averaged responses at $R e=$ $2.3 \times 10^{5}$. The amplitude of the flap oscillations is $10^{\circ}$.


FIGURE 6. (Colour online) Effect of the harmonic forcing on the drag and average base pressure for varying forcing amplitudes. The free-stream velocity is $15 \mathrm{~m} \mathrm{~s}^{-1}$.

Above $S t_{W} \approx 10^{-1}$ the response increases again as the antisymmetric forcing begins to interact with the lateral vortex shedding, leading to a sinusoidal response.

While insensitive to $R e$, figure 6 demonstrates that the magnitude of the frequency response varies significantly with forcing amplitude. This is indicative of nonlinearity in the response between flap angle and pressure measurement. Figure 6 also shows the influence of the forcing on the drag and base pressure, each plotted as a ratio relative to the case with stationary flaps. 
Despite their differences, the frequency responses of figure 6 demonstrate the same general behaviour, consisting of a large response at low frequencies that decays before the interaction with vortex shedding at $S t_{W} \approx 0.2$. This is corroborated by the changes in mean drag and base pressure, also displayed in figure 6. At very low frequencies, there is generally a small drag increase, indicating that regular flipping of the symmetry-breaking mode does not significantly increase the drag compared with the random flipping of the unforced case. By contrast, at higher frequencies, $S t_{W} \gtrsim 0.1$, the drag is seen to rise significantly as the flaps amplify the vortex shedding of the wake. For a range of intermediate frequencies and for small forcing amplitudes, drag reductions of up to $1 \%$ are observed, accompanied by a base pressure increase of $1.7 \%$.

From the phase response shown in the lower part of figure 5(a), we first observe that at all frequencies the output $r$ lags the actuation. While some portion of the phase lag may be attributable to the dynamics captured by the magnitude response, some portion may be due to the advective delay. The monotonic decrease of phase angle with frequency over a region in which the magnitude response is flat $\left(S t_{W} \lesssim 2 \times 10^{-2}\right)$ suggests that such a delay is present. Since the phase responses are independent of $R e$, this delay is observed to be constant in a dimensionless sense, i.e. $\tau U_{\infty} / W$ is constant. This suggests that $\tau$ is an advection time associated with the shear layer perturbations generated by the flap.

A final key observation is the sharp trough seen in some of the frequency response amplitudes at $S t_{W} \approx 0.1$, shown in figures $5(a)$, 6. For a linear transfer function this corresponds to the presence of right half-plane zeros, and is often caused by a cancellation between dynamics operating at different time scales (for the meaning of this, see for example Skogestad \& Postlethwaite 2005). Interestingly this feature is distinct only for large-amplitude forcing and does not correspond to any particular response of the drag or base pressure. It is therefore likely to be a result of cancellation between the measurements of different flow features, rather than the result of any specific flow phenomenon.

\subsection{Offset response}

In addition to the frequency response discussed above, the flaps can also provide a steady forcing: the flaps are placed at a fixed angle and their effect on the distribution $P(r)$ is measured. Figure 7(a) shows the steady state PDF for a number of different flap angles at a free-stream velocity $U_{\infty}=15 \mathrm{~m} \mathrm{~s}^{-1}$. The data show that the flaps induce approximately equal and opposite skewness as they are moved from positive to negative offsets. At a zero offset the PDF is approximately symmetric, with any asymmetry resulting from either imperfect alignment or insufficient averaging time.

Figure 7(b) shows the particular relationship between $\theta$ and the skewness of the $\mathrm{PDF}$, defined here as the natural logarithm of the ratio $P\left(r_{e}\right) / P\left(-r_{e}\right)$. The results demonstrate that a linear fit can be obtained for a range of angles. While more details are provided in $\S 3.5$, the implication of this is that $\theta$ has a linear influence in (3.3), justifying the choice of a term $b \theta_{t-\tau}$.

\subsection{Parameter extraction}

As detailed below, the parameters of the model may now be estimated. The time scales $\alpha$ and $\tau$ can be non-dimensionalised using the body width $W$ and free-stream velocity $U_{\infty}$. The remaining parameters $\lambda, \sigma^{2}$ and $b$ can all be written as ratios with respect to $\alpha$, thereby showing the relative importance of the terms in (3.3). However, 

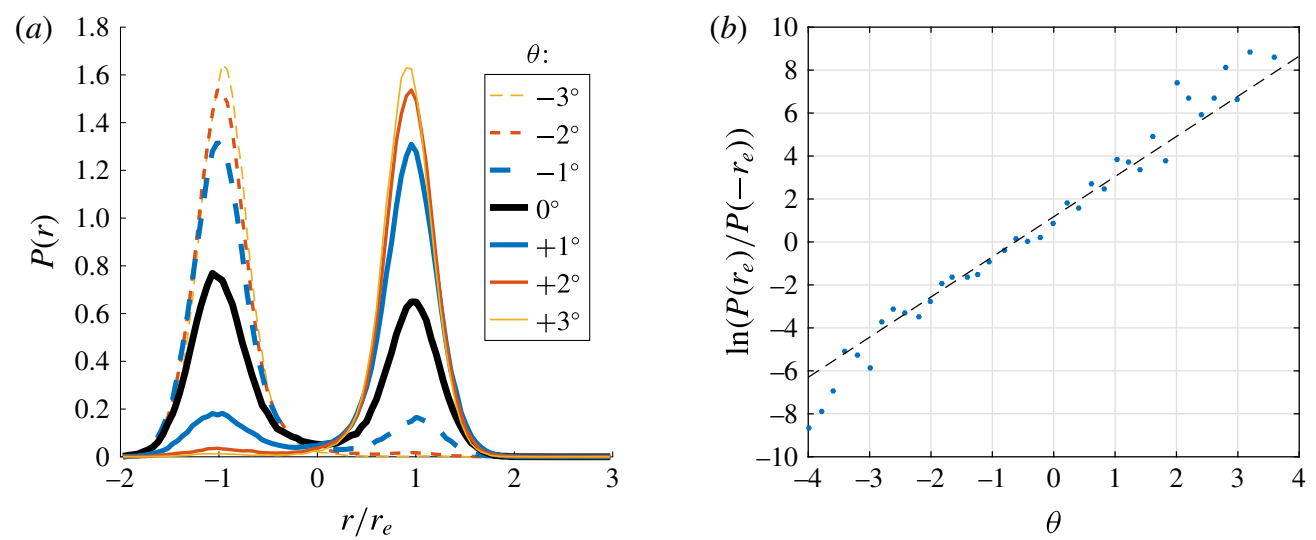

FIgURE 7. (Colour online) (a) Steady state PDF $P(r)$ under a range of steady flap angles $\theta$ and $(b)$ skewness as a function of $\theta$. The dashed line in $(b)$ shows the linear fit used to establish the parameter $b$.

$$
\begin{array}{cccccc} 
& \frac{\alpha W}{U_{\infty}} & \frac{\tau U_{\infty}}{W} & \frac{\lambda r_{e}^{2}}{\alpha} & \frac{\sigma^{2}}{\alpha r_{e}^{2}} & \frac{b}{\alpha r_{e}} \\
\text { Value } & 0.05 & 1.7 & 1 & 0.23 & 0.11
\end{array}
$$

TABLE 1. Dimensionless parameters for the low-dimensional model (3.3).

the ratio of these parameters is dependent upon the range of values that the mode $r$ takes. Furthermore, the choice of metric for $r$ is somewhat arbitrary; for example, the CoP location could be replaced by the pressure gradient on the base or barycentre of velocity deficit in the wake. Any of these metrics quantify the same flow feature but give different numerical values for the mode $r$. A characteristic value such as the equilibrium value $r_{e}=\sqrt{\alpha / \lambda}$ may therefore be taken into account in the normalisation. The correct method for doing this can be seen by considering the change of variables $q=r / r_{e}$. Under this change (3.3) becomes

$$
\begin{aligned}
\dot{q} & =\frac{1}{r_{e}}\left(\alpha r_{e} q-\lambda r_{e}^{3} q^{3}+b \theta_{t-\tau}+\sigma \xi(t)\right) \\
& =\underbrace{\alpha}_{\alpha^{\prime}} q-\underbrace{\lambda r_{e}^{2}}_{\lambda^{\prime}} q^{3}+\underbrace{\frac{b}{r_{e}}}_{b^{\prime}} \theta_{t-\tau}+\underbrace{\frac{\sigma}{r_{e}}}_{\sigma^{\prime}} \xi(t) .
\end{aligned}
$$

Under the change of variables, each of the model parameters can be seen to be replaced by a normalised version, denoted by the '. As expected, the time scale defined by $\alpha$ remains unchanged but the other terms now depend upon $r_{e}$. This gives an appropriate method for non-dimensionalising the parameters, as shown in table 1 .

The first time scale, $\alpha$, is estimated from the frequency response of figure $5(a)$. We stress here that, while the frequency response is usually used as a method for the identification of linear systems, the behaviour seen here is nonlinear, as may be observed from the first phase average shown in figure $5(b)$ and the variation shown in figure 6 . We therefore do not seek to fit a linear model to obtain $\alpha$, but are still 
able to use the response. The instability time scale associated with $\alpha$ is estimated as the inverse of the corner frequency, seen at $S t_{W} \approx 5 \times 10^{-2}$ in figure $5(a)$. That is, $\delta \approx 1 / \alpha \approx W / 0.05 U_{\infty}$. This gives a value of $\delta \approx 0.2 \mathrm{~s}$ for a free-stream velocity of $15 \mathrm{~m} \mathrm{~s}^{-1}$. Such a time scale is sufficiently small that, when a long time series is viewed, flipping events appear to be instantaneous. However this is notably slower than the period of the vortex shedding: for the same $U_{\infty}$, this period is $\approx 0.07 \mathrm{~s}$.

Secondly, the advective time delay is estimated via the slope of the phase response; the precise method for doing this described in the appendix. The value of this parameter, $O\left(W / U_{\infty}\right)$, is consistent with the interpretation that $\tau$ is associated with the streamwise advection of perturbations generated by the flaps. The parameters $\lambda$ and $\sigma$, quantifying, respectively, the saturation and noise intensity, are estimated from the shape of the PDF shown in figure 4(a). As stated above, $\lambda / \alpha$ determines the location of the peak while $\sigma^{2} / \alpha$ determines the width of the distribution.

Estimation of the parameter $b$ can be found by examining the Fokker-Planck prediction, (3.5). As can be seen, only the term involving $\theta$ gives an effect that is asymmetric about $r=0$. Taking the natural logarithm of the ratio of $P\left( \pm r_{e}\right)$ we obtain

$$
\ln \left(\frac{P\left(r_{e}\right)}{P\left(-r_{e}\right)}\right)=\frac{4 b \theta r_{e}}{\sigma^{2}} .
$$

As shown in figure $7(b)$, the left-hand side of this equation is seen to be linear with respect to $\theta$, within a certain range, enabling $b$ to be estimated by the slope of the fit.

The dimensionless form for $\lambda$ is by definition equal to unity, while those for $\sigma$ and $b$ are seen to be of $O(0.1)$. The relative size of $\lambda$ and $\sigma^{2}$ is therefore consistent with the fairly sharp peaks in the PDFs of figures $4(a)$ and $7(a)$. Given flap angles $\theta$ of $O\left(10^{\circ}\right)$, the $b \theta$ term will be large relative to the noise and instability terms, suggesting that the flaps have good control authority.

\section{Feedback control}

The model (3.3) for the RSB mode describes a pitchfork bifurcation. In agreement with the suggestion of Cadot et al. (2015), there is therefore an unstable equilibrium corresponding to a symmetric flow. Furthermore, the model is seen to be valid over a range of $R e$, justifying its use in the design of linear controllers that try to recover this unstable equilibrium. It is important to note that unlike those scenarios in which the suppression of unsteadiness is the key control goal (e.g. Dahan et al. 2012), here we seek to stabilise a steady bifurcated mode. That is, we seek to change the equilibrium position and therefore the steady behaviour of the closed-loop system. A schematic of the feedback control loop is shown in figure 8 .

\subsection{System formation}

Here, we form the transfer functions that appear in figure 8 for the plant, $G_{1,2}$, and actuator, $A$. As will be detailed further below, the wake is considered to comprise the sum of the bistability dynamics $\left(G_{1}\right)$ and those of the shear layers and vortex shedding occurring at higher frequencies $\left(G_{2}\right)$.

Using the model and results shown in $\S 3$ we can form the linear model $G_{1}$ of figure 8. The model described by (3.3) is a nonlinear time-delayed differential equation. In order to design a linear controller we first linearise about the desired equilibrium point of $r=0$, which essentially eliminates the $\lambda r^{3}$ term. This is 


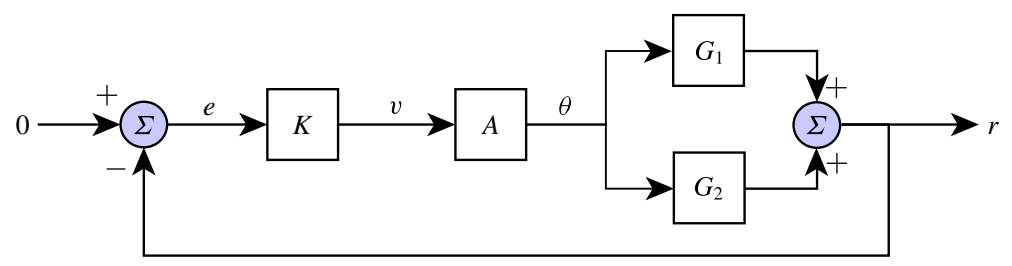

FIGURE 8. (Colour online) A schematic of the feedback control loop comprising the wake $G_{1,2}$, actuator $A$ and feedback controller $K ; G_{1}$ captures the bistability dynamics while $G_{2}$ captures the vortex shedding and shear layer dynamics at higher frequencies. The controller aims to make the metric $r$ equal to zero in order to achieve a symmetric wake.

unproblematic since this term provides a saturation so is likely to help the control action. Moreover, the controller will be aiming to make $r$ small, and thereby achieve a flow state in which this term is negligible. Secondly, having transformed the system to the Laplace domain, we use a first-order Padé approximation for the time delay (Åström \& Murray 2008). This gives the following transfer function between $\theta$ and $r$ :

$$
G_{1}(s)=\frac{\bar{r}(s)}{\bar{\theta}(s)}=\frac{b(2 / \tau-s)}{(s-\alpha)(2 / \tau+s)},
$$

where $s$ is the complex Laplace transform variable. This transfer function has one unstable pole at $s=\alpha$ corresponding to the flow instability, as well as a pole and zero at $s= \pm 2 / \tau$, resulting from the time delay. The presence of the unstable pole and right half-plane zero both imply that control of the system will have limitations (Skogestad \& Postlethwaite 2005); in particular this implies that we cannot hope to have control authority over a wide frequency range.

In addition to the transfer function specifying the dynamics of the bistability, it is insightful to capture the dynamics present at higher frequencies. This dynamics is evident for $S t_{W}>0.1$, as shown in figure 5(a). Unlike for the RSB mode, we do not have an a priori model describing the dynamics at these frequencies. Instead we simply seek to fit a linear model that captures the necessary amplitude and phase information, enabling us to take this into account in the control design. Our fit to the higher-frequency dynamics is shown in figure 9. As shown, we only fit the response for $S t_{W}>0.11$. Below this frequency the bistability dynamics is dominant, and we therefore do not require a linear fit to the (nonlinear) dynamics. For $S t_{W}>0.11$ the response of the flow to open-loop forcing is much more sinusoidal than at lower frequencies (figure 5b) and a linear fit such as that applied in (Dahan et al. 2012) may be appropriate. We emphasise that the purpose of this is simply to understand any interactions between the controller and the higher-frequency dynamics; it is (4.1) that captures the key information about the dynamics of the RSB mode.

Finally it is necessary to identify $A$, the dynamics of the actuator. This is important since the finite bandwidth of the actuator, and the limitations this imposes, must be included in the control design. As can be seen in figure 9, the actuator acts as a linear oscillator, the properties of which can be tuned by the choice of springs or the addition of masses. We choose the actuator to have a natural frequency corresponding to $S t_{W} \approx 0.24$ at $15 \mathrm{~m} \mathrm{~s}^{-1}$, giving sufficient bandwidth to control the bistability which manifests for $S t_{W} \lesssim 0.1$. It can be seen that the linear second-order model in figure 9 captures the dynamics accurately. 

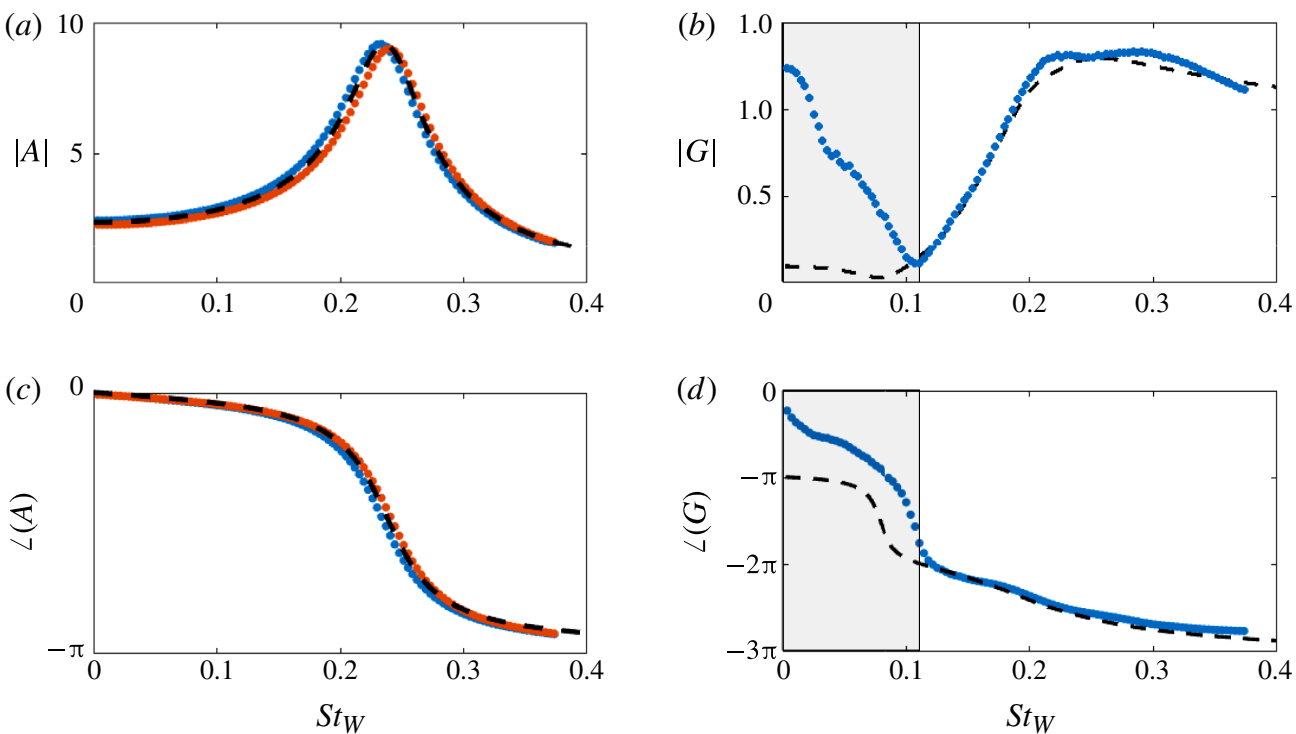

FIgURE 9. (Colour online) Linear fits to the actuator dynamics $(a, c)$ and higher-frequency wake dynamics $(b, d)$. A fifth-order linear fit to the wake dynamics is found using the MATLAB command fitfrd, applied only to the part of the frequency response to the right of the shaded region. A second-order fit is found for the actuator, between an input in volts and an output in degrees.

\subsection{Preliminary control design}

Based purely upon the transfer function $G_{1}$ derived from our model, the system shown in figure 8 has a closed-loop transfer function given by

$$
\begin{aligned}
T(s) & =\frac{A(s) K(s) G_{1}(s)}{1+A(s) K(s) G_{1}(s)} \\
& =\frac{n_{A} n_{K} n_{G}}{d_{A} d_{K} d_{G}+n_{A} n_{K} n_{G}}
\end{aligned}
$$

where $n$ and $d$ respectively denote the numerator and denominator of the individual transfer functions. The stability, among other closed-loop properties, is determined by the roots of the denominator of $T(s)$ (denoted by $d_{T}(s)$ ). For an initial analysis we can consider the case without actuator dynamics, i.e. $A=a_{0}$, a static gain. For $K=k_{p}$, a simple proportional gain, we have

$$
d_{T}(s)=s^{2}+\left(\frac{2}{\tau}-k_{p} b a_{0}-\alpha\right) s+\frac{2}{\tau}\left(k_{p} b a_{0}-\alpha\right) .
$$

All variables here have the same meaning as in (4.1). For stability, the Routh-Hurwitz criterion requires that each of the bracketed terms be positive (Åström \& Murray 2008). This requires that

$$
\frac{\alpha}{b a_{0}}<k_{p}<\frac{2-\tau \alpha}{\tau b a_{0}} .
$$

There is therefore expected to be a gain margin, just as there is for the control of vortex shedding over cylinders (Illingworth, Naito \& Fukagata 2014). A necessary 


$\begin{array}{lccccc}\text { Controller } & k_{i} & \Delta C_{D}(\%) & \left\langle\Delta C_{p}\right\rangle(\%) & P_{a c t}(\%) & S t_{\max } \\ \text { Open loop } & - & -1.0 & 1.7 & - & - \\ K_{1} & 0.07 & -0.9 & 1.7 & 11 & 0.22 \\ K_{2} & 0.13 & -1.6 & 3.7 & 15 & 0.19 \\ K_{3} & 0.20 & -2.0 & 3.9 & 24 & 0.13\end{array}$

TABLE 2. The controllers and their performance including the controller gain, drag reduction, base pressure change, actuation power as a percentage of the power saving and the most amplified frequency. For comparison, the results for the open-loop forcing giving maximal drag reduction are also shown.

condition for the existence of a $k_{p}$ satisfying (4.4) is therefore

$$
\alpha \tau<1 .
$$

This makes intuitive sense because if the time delay is equal to, or greater than, the time scale of the RSB instability, the feedback system will be unable to respond quickly enough. Inspection of the values in table 1 confirms that this condition is satisfied and therefore that, in the absence of other wake dynamics, the bistable wake mode can be stabilised using proportional feedback.

\subsection{Detailed control design and performance}

Given the models for $G(s)$ and $A(s)$ we can now design controllers, $K(s)$. We present the results here from three controllers of increasing complexity. In each case the form of the controller is chosen before the gain is manually tuned to identify the condition for maximum drag reduction. Each controller also features a low-pass filter $\left(K_{L P}\right)$ with a $3 \mathrm{~dB}$ frequency corresponding to $S t_{W} \approx 10$, intended to filter out noise in order to reduce power consumption. The properties and results from the three controllers are summarised in table 2 .

\subsubsection{Proportional controller, $K_{1}$}

Based upon the above analysis, a first controller was chosen to be of the form

$$
K_{1}(s)=k_{1} \underbrace{\frac{1}{1+s T_{L}}}_{K_{L P}},
$$

where $T_{L}$ is the time constant of the low-pass filter $K_{L P}$. The optimal gain $k_{1}$ with respect to drag reduction was found by manual tuning. It can be seen from table 2 that such a controller achieves a drag reduction of $0.9 \%$ and requires only $11 \%$ of the saved power in order to drive the actuators. By comparing the curves for $K_{1}$ and the uncontrolled case in figure 10(a), it can also be seen that the controller makes the wake more symmetric on average, i.e. the mode of the PDF has moved to a smaller value of $r$.

The limitations of the proportional controller can be seen by looking at the sensitivity function $S(s)=1 /(1+A(s) K(s) G(s))$. This is shown in figure $11(b, d)$. For a stable system, the sensitivity function gives the expected ratio of fluctuations with and without control; therefore values of greater than unity indicate that the controller 

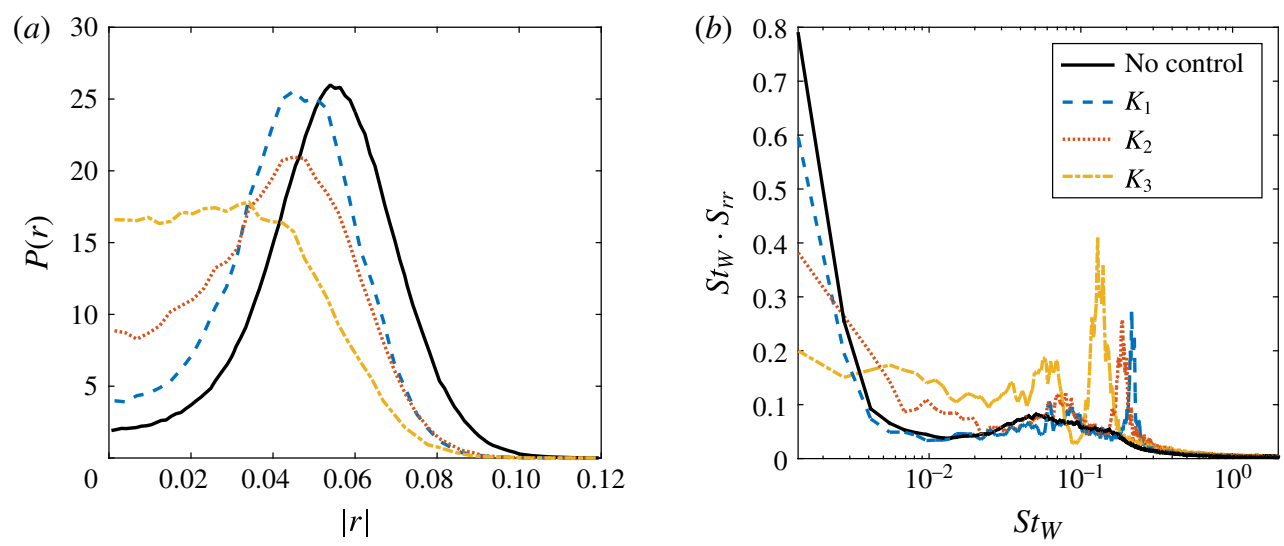

FIGURE 10. (Colour online) PDF for $r(a)$ and premultipled spectra $(b)$ under the three controllers. The solid black line shows the uncontrolled case for comparison.
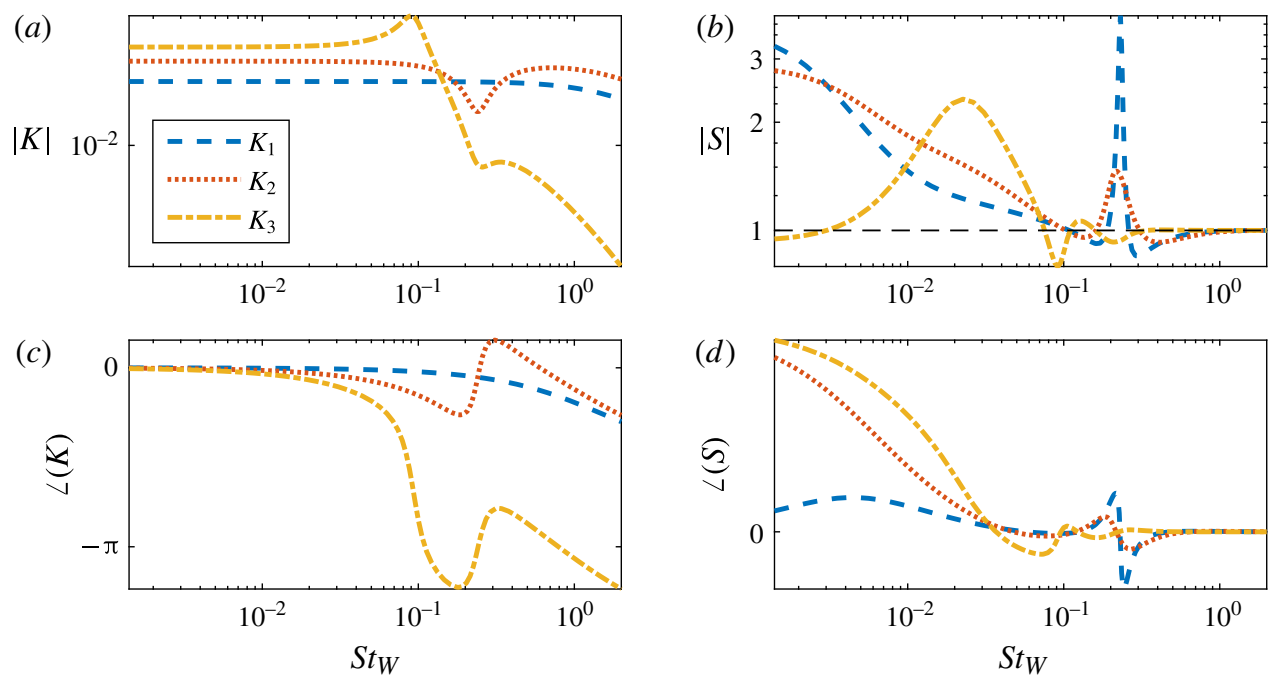

FIGURE 11. (Colour online) Bode plot for the controllers $(a, c)$ and the expected sensitivity function $S(b, d)$.

amplifies fluctuations. It can be seen that for $K_{1}$ there is a large peak at $S t_{W} \approx 0.23$. The prediction of the sensitivity function is borne out in the closed-loop PSD, shown in figure $10(b)$, where there is evidently a large peak in the fluctuations at $S t_{W} \approx 0.22$. The open-loop results displayed in figure 6 demonstrate that fluctuations at this frequency lead to a large drag increase, and most likely correspond to an interaction with the vortex shedding. Since any further increase in gain increases the magnitude of these fluctuations, the optimal gain is found as the trade-off between providing increasing suppression of the bistability and avoiding this undesired interaction.

\subsubsection{Filtered controller, $K_{2}$}

The limitation of the proportional controller arises in part from the actuator dynamics: the peak sensitivity coincides with the resonant frequency of the actuator, 


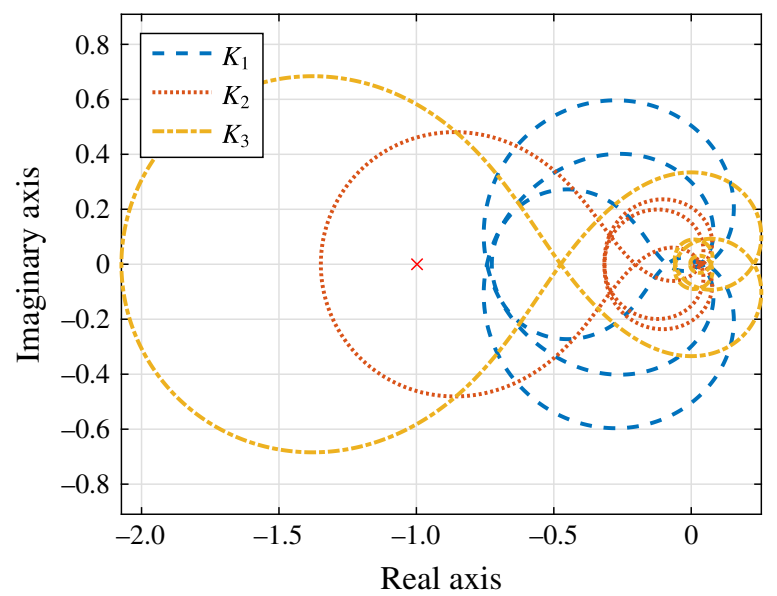

FIGURE 12. (Colour online) The Nyquist diagram for the three controllers. For stabilisation of the RSB mode we require one encirclement of the -1 location, as marked by the red cross.

as seen in figure 9. This dynamics may be catered for by the inclusion of a notch filter $\left(K_{N}\right)$ that counteracts the resonant behaviour, giving a controller of the form

$$
K_{2}(s)=k_{2} \underbrace{\frac{1+2 \zeta_{n} s T_{A}+s^{2} T_{A}^{2}}{1+2 \zeta_{d} s T_{A}+s^{2} T_{A}^{2}}}_{K_{N}} \underbrace{\frac{1}{1+s T_{L}}}_{K_{L P}},
$$

where $\zeta_{n}<\zeta_{d}$ and $T_{A}$ is the time constant of the actuator. The controller may again be tuned by minimising the drag with respect to the gain $k_{2}$. The optimal drag reduction and base pressure increase, shown in table 2 , are significantly increased to $1.6 \%$ and $3.7 \%$ respectively, while the control is only marginally less efficient than for $K_{1}$. Figure 10(a), demonstrates that bistability is suppressed further relative to the $K_{1}$ case since the peak in the PDF is reduced.

As in the previous control case, the limitation to the gain comes from fluctuations occurring at higher frequencies. This is consistent with the sensitivity function shown in figure $11(b, d)$, which predicts amplification at $S t_{W} \approx 0.22$, and is also confirmed by the spectra in figure $10(b)$, which shows a distinct peak for $S t_{W} \approx 0.19$. The controller again represents a trade-off between achieving sufficient gain to stabilise the RSB mode and avoiding amplification of high-frequency fluctuations that are detrimental to the drag reduction.

\subsubsection{Loop-shaped controller, $K_{3}$}

The previous two controllers are both limited by the presence of fluctuations at higher frequencies that are detrimental to the drag. These fluctuations limit the gain that can be achieved at lower frequencies, thus imposing a limitation on the extent to which the bistability can be suppressed. In order to improve this, it is insightful to look at the Nyquist diagram for each controller, as shown in figure 12.

The Nyquist diagram plots the real and imaginary parts of the loop transfer function $L(s)=G(s) A(s) K(s)$, and can be used to determine the expected stability and robustness. In order to stabilise the instability that leads to the RSB mode, 
one encirclement of the -1 point on the Nyquist diagram is required (Åström \& Murray 2008). Practically, this means that the static gain must be sufficiently large, as discussed in $\S 4.2$. It can be seen from figure 12 that $K_{1}$ does not achieve an encirclement while $K_{2}$ does. The nonlinear saturation means that an encirclement is not strictly necessary in order for a positive effect to be achieved, but the Nyquist curves still help explain why greater suppression of the asymmetry is achieved with $K_{2}$.

The Nyquist diagram also gives insight into the sensitivity function: since $S(s)=$ $1 /(1+L(s))$, the sensitivity at any given frequency is determined by the distance between the Nyquist curve at that frequency and the -1 point (shown by the cross). Minimising the sensitivity function therefore necessitates keeping the Nyquist curve as far from the -1 location as possible. However, it is also important to understand that there are fundamental limitations to the extent to which this can be achieved. A well-known limitation comes from Bode's sensitivity integral,

$$
\int_{0}^{\infty} \log |S(\mathrm{i} \omega)| \mathrm{d} \omega=\pi \sum p_{k},
$$

where $p_{k}$ are the unstable poles of $L(s)$. In our case $\pi \sum p_{k}=\pi \alpha$. Therefore equation (4.8) means that while the sensitivity function may be designed to be small at some frequencies, over some other frequency range fluctuations must be amplified by the controller. In terms of drag reduction, it is important to try to place these fluctuations in a frequency range that has the least negative effect on the drag. This frequency range is found from the open-loop forcing and shown in figure 6.

The final loop-shaped controller can be seen in figure 11(a,c), and the 'loop' seen on the Nyquist diagram of figure 12. The controller consists of the same notch filter as $K_{2}$ but with the addition of a second-order resonant filter $\left(K_{R}\right)$, specified by the parameters $\zeta_{3}$ and $T_{3}$. The objective of this filter is to reduce the loop gain over the frequency range in which vortex shedding is amplified, while minimising the phase lag at lower frequencies. This shaped controller is given by

$$
K_{3}(s)=k_{3} \underbrace{\frac{1}{1+2 \zeta_{3} s T_{3}+s^{2} T_{3}^{2}}}_{K_{R}} \underbrace{\frac{1+2 \zeta_{n} s T_{A}+s^{2} T_{A}^{2}}{1+2 \zeta_{d} s T_{A}+s^{2} T_{A}^{2}}}_{K_{N}} \underbrace{\frac{1}{1+s T_{L}}}_{K_{L P}} .
$$

The loop shape can be seen to be improved by the wider encirclement of the -1 location, indicating that the low-frequency gain and phase are appropriate. This is consistent with the sensitivity function of figure $11(b, d)$, which is seen to demonstrate a much smaller peak and at a lower frequency compared to the previous two controllers.

As shown in table 2, the loop-shaped controller $K_{3}$ gives the highest drag reduction and base pressure increase as $2.0 \%$ and $3.9 \%$, while also maintaining good energy efficiency. From figure $10(a)$, it is clear that the bistability has now been almost entirely suppressed as there is no longer a peak present in the PDF. This can be attributed to the higher gain of the controller, made possible by the loop shaping. Conversely, it can be seen from figure $10(b)$ that $K_{3}$ led to the largest energy fluctuations of all the controllers, but was still able to achieve the highest drag reduction. This was done by placing these fluctuations at lower frequencies at which their effect on the drag is smaller, as demonstrated from the results in figure 6.

\subsection{The controlled flow}

Having observed that the controller may achieve up to a $2 \%$ drag reduction, it is worth assessing the changes that the controller makes to the flow. This may first be 


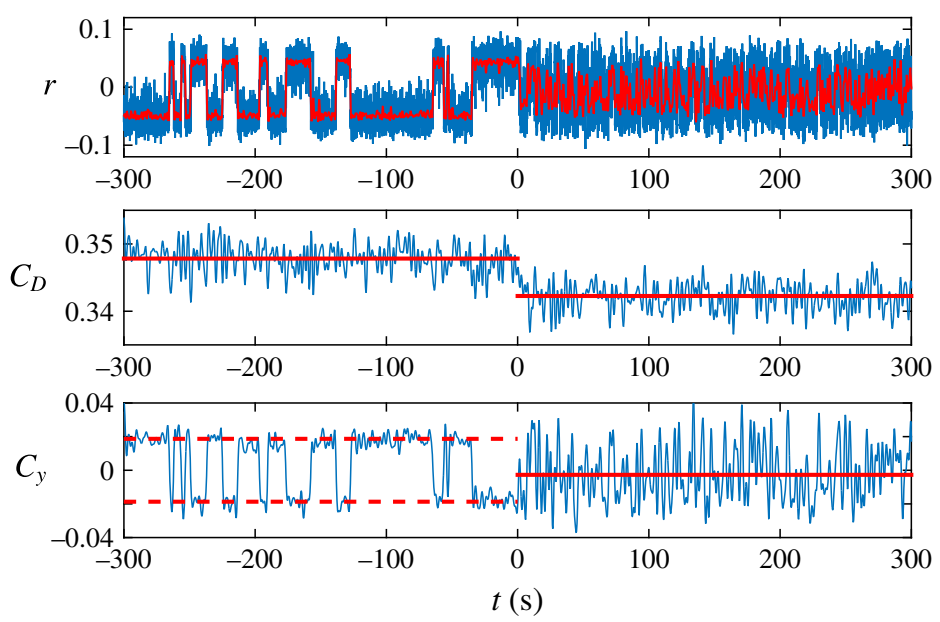

FiguRE 13. (Colour online) Time series of the mode $(r)$, drag coefficient $\left(C_{D}\right)$ and lateral force coefficient $\left(C_{y}\right)$. The controller is turned on at $t=0$. Red lines show the low-pass filtered $r$ and mean forces over the uncontrolled and controlled periods.

done by examining the time series of the mode $r$, as shown in figure 13. The complete time series shows data before and after the controller is turned on at $t=0$. For $t<0$ $r$ is seen to flip randomly, as is typical for the natural flow. With the application of the control there is an abrupt change in the behaviour, the random flipping ends and smaller values of $r$ become much more probable, as also indicated in the PDF shown in figure $10(a)$.

Figure 13 also displays the drag coefficient $C_{D}$ and lateral force coefficient $C_{y}$. It is evident that with the application of control there is an almost immediate reduction in $C_{D}$. This reduction is seen to be accompanied by a significant change in the lateral force. For $t<0, C_{y}$ is seen to switch between two equal and opposite extremes as a result of the bistable wake. The average size of this lateral force is shown by the dashed lines. For $t>0$ the bimodal behaviour is suppressed and the average magnitude of the lateral force is significantly reduced, although the fluctuations are still significant. While the magnitude of the lateral force is shown here, it is important to note that the drag is likely related to the square of the lateral force $\left\langle C_{y}^{2}\right\rangle$, due to induced drag effects (Grandemange et al. 2013a, 2014b). However, a quantitative evaluation of $\left\langle C_{v}^{2}\right\rangle$ may not be made from this experiment, as the inertial forces associated with the flap motion provide a significant fluctuating contribution to $C_{y}$.

We may also assess the motion of the flaps, in order to deduce the mechanism by which the controller is able to reduce the drag and lateral forces. Figure 14(a) shows a snapshot of the time series of $r$ and $\theta$. It is clear from the time series that the two signals are closely linked, resulting from the proportional action of the controller. It can be seen that as the mode moves towards one extreme (e.g. at $t \approx$ $29,32 \mathrm{~s}$ ), the flaps also move in order to compensate, thereby causing the mode to move back towards a value of zero. The reactive motion of the flaps leads to a broadband spectrum for their motion, as displayed in figure 14(b). This indicates that the flaps do not achieve suppression of the bistability via a quasi-open-loop effect. Rather, they rely on the nature of a true feedback control system.

Although the time series of $r$ and $\theta$ look superficially similar, the spectra demonstrates some key differences, principle among which is the peak in $S_{r r}$ at 
(a)
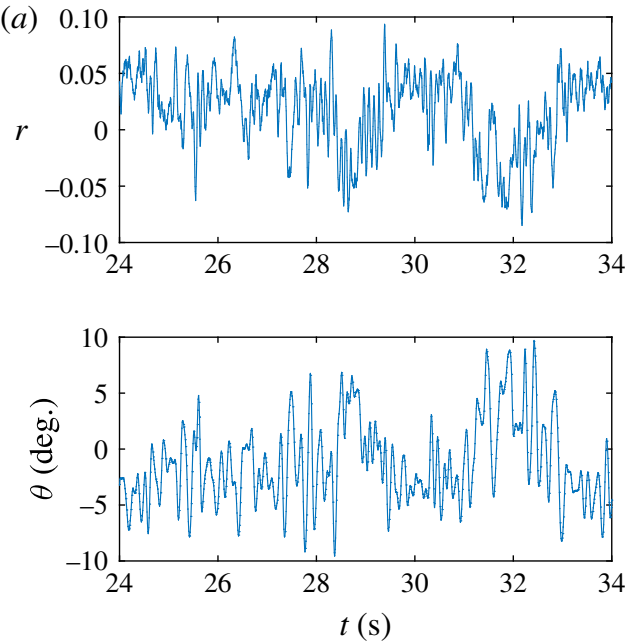

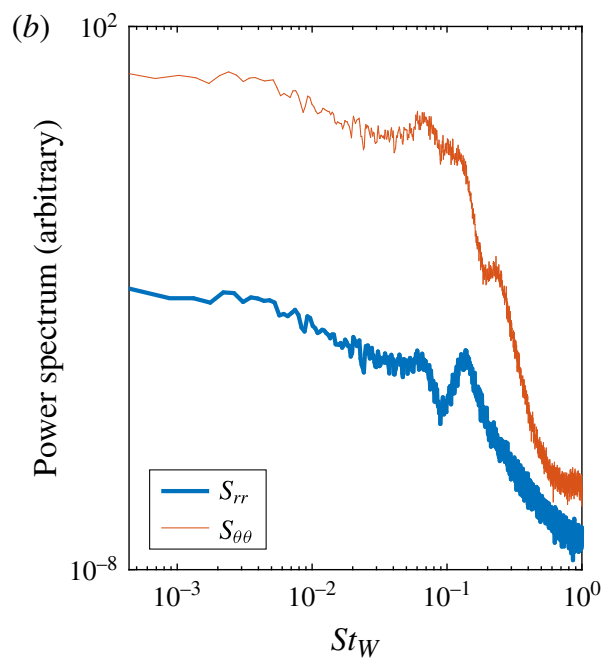

FIgURE 14. (Colour online) The dynamics of the actuator showing $(a)$ a short time series of the mode $r$ and flap angles $\theta$, and $(b)$ the corresponding power spectra.

$S t_{W} \approx 0.13$, just as displayed in figure $10(b)$. As noted above, this peak is predicted by the sensitivity function displayed in figure $11(b, d)$, and is therefore a result of the interaction between the flaps and the wake, arising due to the feedback. While we do not have velocity measurements to confirm the exact nature of these oscillations, it is likely that they result from an interaction with the vortex shedding of the wake since both the forcing and the measurement are antisymmetric, just as for a von Kármán vortex street. Furthermore, the open-loop results shown in figure 6 demonstrate that open-loop forcing at $S t_{W}=0.13$ leads to a small drag increase irrespective of the amplitude. It is therefore reasonable to expect that these oscillations in the closed-loop system act to increase the drag on the body and that if they could be avoided the drag reduction may be improved.

Finally, it is important to note that the measurements here cannot distinguish drag reduction from any thrust produced by the flaps. Such thrust could manifest as a force directly on the flaps or as a pressure force on the body. However, any thrust-producing mechanism would have negative efficiency: the power required would be greater than the power saving. The efficiency of the system detailed in table 2 therefore suggests that the primary drag reduction mechanism is not thrust generation.

\section{Discussion and conclusions}

\subsection{The nature of the bistability}

As we have seen in $\S 3$, the bistability observed in 3-D bluff body wakes can be modelled fairly accurately by a nonlinear Langevin equation. This can be seen not only from the time-averaged behaviour in figure $4(a)$ but also from the qualitative description that (3.3) gives: that of a wake flipping between two stable equilibrium positions, perturbed by the noise arising due to turbulence. While it cannot be stated that the model is more than a convenient approximation, we may still derive from it insight concerning the dynamics of the process and the application of feedback control.

The model of (3.3) can be seen to be consistent with observations of the flow over different Reynolds numbers, both in the unperturbed case and under forcing with 
the flaps. We find that the PDF of the mode $r$ exhibits only a slight trend with $R e$, indicating that the topology of the RSB mode remains almost constant. Furthermore, the forced results of figure $5(a)$ indicate that the dimensionless time scales of the process also remain constant with changing $R e$. Neither of these observations may be surprising, since the large-scale structures are dominated by inertial forces that should be more or less independent of viscosity.

The bistability may be quantified in terms of the time scales $\delta$ and $T$, respectively, the instability time scale and the flipping period. We have demonstrated here through the use of forcing flaps that the former is finite and notably larger than the vortex shedding period. Our model suggests that the ratio of these time scales may be related to the ratio $\sigma^{2} / \alpha$, which the experimental results demonstrate to be roughly constant. This indicates that any increase in the growth rate $\alpha$ is accompanied by a proportionate change in the noise intensity $\sigma^{2}$. Our model and observations are consistent with those of Grandemange et al. (2013c), who found a linear increase in the flipping rate with increased free-stream velocity, corresponding to a reduction in the time scale $T$ and therefore an increase in $\sigma^{2}$. Since we find that $\alpha \propto U_{\infty}$, this increased flipping rate is accompanied by an increased growth rate and therefore the ratio $\sigma^{2} / \alpha$ may remain constant with changing $R e$.

Finally, the feedback control implementation reinforces the results of Cadot et al. (2015), who show that suppression of bistability can lead to a drag reduction. We find that by reducing the asymmetry of the wake, as measured by the base pressure, the drag may be reduced by as much as $2 \%$. Furthermore, this coincides with suppression of the mean magnitude of the lateral force (but not its fluctuations), and as suggested by Grandemange et al. (2013c) confirms the link between the lateral and streamwise forces. Finally, the idea that the bistability may be controlled by proportional feedback provides a possible explanation for the suppression achieved via a control cylinder (Cadot et al. 2015), and may give insight into other passive techniques.

\subsection{Implications for feedback control design}

As seen in $\S 4.1$, the present model can be transformed into a system for which a feedback controller can be designed. This is demonstrated to be an energetically efficient way to reduce the pressure drag on a 3-D bluff body. The model and results suggest that an adequate proportional feedback is required for good suppression of the instability leading to bistability. However, the results also suggest that other dynamics must be taken into account if an effective controller is to be designed. In particular, a linear fit to the frequency response around the frequencies of the vortex shedding and shear layers may be used to adjust the dynamics of the controller. In this way fluctuations may be placed at frequencies at which their negative impact is minimised, and an adequate proportional feedback may be achieved.

A key feature in terms of achieving efficiency is the choice of actuator. The flaps used here provide forcing on the wake by deflecting the shear layers either inwards or outwards. In this way a lateral momentum flux can be generated by the flaps with little energy input. This is in contrast to actuators such as massless jets in which the momentum flux is generated directly by the actuator and therefore requires direct energy input. Because the flaps used here consist of a mass-spring system, minimising their power consumption requires a trade-off against control efficacy. Much of the power consumption arises due to inertial loads and the work done in deforming the springs: low power consumption therefore requires a low mass and soft springs. This is in contrast to the requirements of the control for which a stiff spring is required to maximise the actuator bandwidth. 
Also key for control design is the choice of sensors used for feedback. We have demonstrated here that the use of only six suitably positioned base pressure sensors may be adequate for feedback control. Moreover, it is likely that the same control may be achieved with as few as two sensors, albeit at the expense of a noisier measurement.

Despite the demonstrated efficacy of the controller, it is possible that improvements may be made with a different arrangement of sensors. For the unforced case, base pressure measurements perform well as they pick up the strong asymmetry imposed by the recirculation bubble. However, as seen from the frequency response in figure $5(a)$, there is a frequency at which no response is detected. Furthermore, this feature is indicative of non-minimum-phase behaviour of the system, as indicated by the abrupt decrease in phase angle that accompanies the low response. Such behaviour is often due to the specific choice of sensor type and location (Åström \& Murray 2008), and will always impose control limitations (Skogestad \& Postlethwaite 2005). It is therefore possible that future improvements may be achieved with a different sensor configuration.

\subsection{Concluding remarks}

In this paper we have demonstrated that the RSB mode of rectilinear bluff body wakes can be modelled by a nonlinear Langevin equation, in which the deterministic part describes the evolution of the large-scale structure and the stochastic part models the influence of the turbulent forcing. The dimensionless model parameters are seen to remain approximately constant with changing $R e$, demonstrating the validity of the approach over a range of conditions. Furthermore, the model can be used in the design of a feedback controller that uses dynamic flaps to try to restore the naturally unstable symmetric flow condition. This feedback controller was implemented experimentally and was demonstrated to both suppress the bistability of the wake and efficiently reduce the drag experienced by the body.

The results shown in this paper appear to be the first demonstration of an efficient feedback control strategy applied to a high-Re 3-D bluff body wake. The results confirm that, given a sufficiently good understanding and suitably positioned actuators and sensors, suppression of large-scale flow structures is a valid method to efficiently reduce the drag of bluff bodies. Furthermore, these results suggest that the equations modelling the laminar large-scale structures provide a suitable starting point for control design. We therefore expect this work to pave the way for future advances, not only in the suppression of the symmetry-breaking mode of 3-D wakes but also in the suppression of other dominant wake features.

\section{Acknowledgements}

R.D.B. acknowledges an Imperial College PhD Scholarship. We are also indebted to EPSRC (grant nos. EP/I005684, EP/K503733/1), the Imperial College EPSRC Impact Acceleration Account, the Climate KIC Accelerator Program and Imperial Innovations for financial support.

\section{Appendix. Time delay estimation}

Estimation of the convective time scale $\tau$ can be achieved from the frequency response shown in figure 15. The magnitude response allows the definition of a linear, minimum-phase system using the fitmagfrd MATLAB function. This fitted 

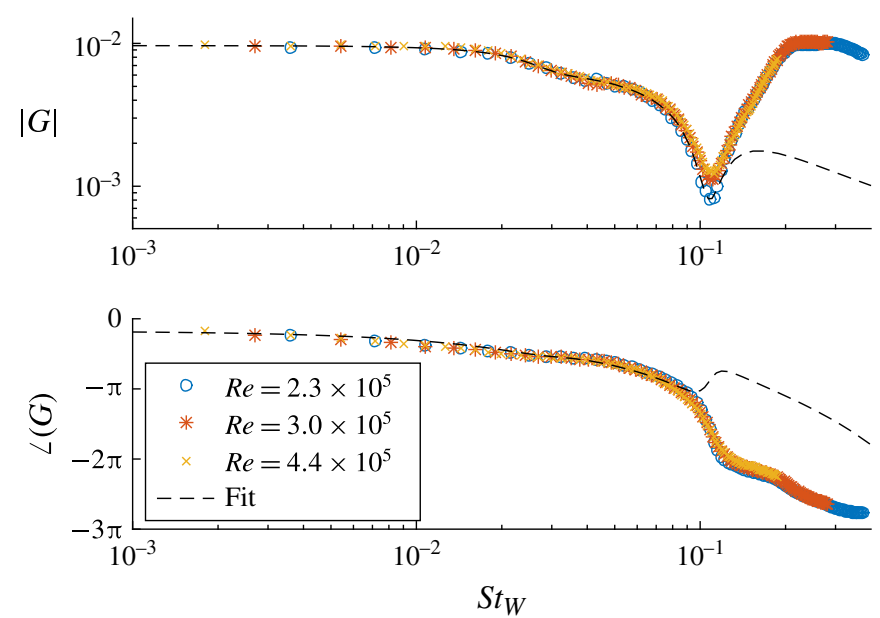

FIgURE 15. (Colour online) The response of the wake shown in figure 5(a) along with the linear fit used to estimate the time delay $\tau$.

system has a phase corresponding to the minimum-phase part of the system. Any remaining phase response can be attributed to the dead zone (evaluated for the lowest-frequency case) and the delay, both of which are non-minimum-phase features. A delay term simply gives a linear decrease in phase angle with frequency while the dead zone gives a constant (negative) phase angle. This delay can simply be adjusted until the phase response of the modelled system matches that of the experiment.

\section{REFERENCES}

Ahmed, S., Ramm, G. \& FAltin, G. 1984 Some salient features of the time-averaged ground vehicle wake. SAE Tech. Rep. 840300, Society of Automotive Engineers.

Åström, K. J. \& Murray, R. M. 2008 Feedback Systems An Introduction for Scientists and Engineers. Princeton University Press.

Barbagallo, A., Dergham, G., SipP, D., Schmid, P. J. \& Robinet, J.-C. 2012 Closed-loop control of unsteadiness over a rounded backward-facing step. J. Fluid Mech. 703, 326-362.

Barbagallo, A., SiPP, D. \& SCHMID, P. J. 2009 Closed-loop control of an open cavity flow using reduced-order models. J. Fluid Mech. 641, 1-50.

BURY, Y. \& JARDIN, T. 2012 Transitions to chaos in the wake of an axisymmetric bluff body. Phys. Lett. A 376 (45), 3219-3222.

CAdot, O., EvRARD, A. \& PASTUR, L. 2015 Imperfect supercritical bifurcation in a three-dimensional turbulent wake. Phys. Rev. E 91, 063005.

Dahan, J. A., Morgans, A. S. \& Lardeau, S. 2012 Feedback control for form-drag reduction on a bluff body with a blunt trailing edge. J. Fluid Mech. 704, 360-387.

Evrard, A., Cadot, O., Herbert, V., Ricot, D., Vigneron, R. \& Délery, J. 2015 Fluid force and symmetry breaking modes of a 3D bluff body with a base cavity. J. Fluids Struct. 61, 99-114.

Fabre, D., Auguste, F. \& Magnaudet, J. 2008 Bifurcations and symmetry breaking in the wake of axisymmetric bodies. Phys. Fluids 20, 051702.

Gammaitoni, L., Hänggi, P., Jung, P. \& Marchesoni, F. 1998 Stochastic resonance. Rev. Mod. Phys. 70 (1), 223-287.

García De la Cruz, J. M., OXlade, A. R. \& Morrison, J. F. 2016 Passive control of base pressure of an axisymmetric blunt body using an interconnected perimetric slit (in preparation). 
Gautier, N. \& Aider, J.-L. 2014 Feed-forward control of a perturbed backward-facing step flow. J. Fluid Mech. 759, 181-196.

Gautier, N., Aider, J.-L., Duriez, T., Noack, B. R., Segond, M. \& Abel, M. 2015 Closed-loop separation control using machine learning. J. Fluid Mech. 770, 442-457.

Grandemange, M. 2013, Analysis and control of three-dimensional turbulent wakes: from axisymmetric bodies to road vehicles. PhD thesis, École Polytechnique - ENSTA ParisTech.

Grandemange, M. A., Mary, G. M. \& CADOt, O. 2013a Effect on drag of the flow orientation at the base separation of a simplified blunt road vehicle. Exp. Fluids 54, 1529.

Grandemange, M., Саdot, O. \& GohlKe, M. 2012 Reflectional symmetry breaking of the separated flow over three-dimensional bluff bodies. Phys. Rev. E 86, 035302.

Grandemange, M., Gohlke, M. \& CAdot, O. 2013b Bi-stability in the turbulent wake past parallelepiped bodies with various aspect ratios and wall effects. Phys. Fluids 25, 095103.

Grandemange, M., GohlKe, M. \& CAdOT, O. 2013c Turbulent wake past a three-dimensional blunt body. Part 1. Global modes and bi-stability. J. Fluid Mech. 722, 51-84.

Grandemange, M., Gohlke, M. \& CAdot, O. 2014a Statistical axisymmetry of the turbulent sphere wake. Exp. Fluids 55, 1838.

Grandemange, M., Gohlke, M. \& CAdot, O. $2014 b$ Turbulent wake past a three-dimensional blunt body. Part 2. Experimental sensitivity analysis. J. Fluid Mech. 752, 439-461.

Henning, L., Becker, R., Feuerbach, G., Muminovic, R., King, R., Brunn, A. \& Nitsche, W. 2008 Extensions of adaptive slope-seeking for active flow control. Proc. Inst. Mech. Engrs 222, 309-322.

Herry, B., Keirsbulck, L., Labraga, L. \& Paquet, J.-B. 2011 Flow bistability downstream of three-dimensional double backward facing steps at zero-degree sideslip. Trans. ASME J. Fluids Engng 133 (5), 054501.

Ho, Y. C. \& PePyne, D. L. 2002 Simple explanation of the no-free-lunch theorem and its implications. J. Optim. Theor. Applics. 115, 549-570.

Illingworth, S. J., Morgans, A. S. \& Rowley, C. W. 2012 Feedback control of cavity flow oscillations using simple linear models. J. Fluid Mech. 709, 223-248.

Illingworth, S. J., NAito, H. \& FukAGata, K. 2014 Active control of vortex shedding: an explanation of the gain window. Phys. Rev. E 90, 043014.

Meliga, P., Chomaz, J.-M. \& Sipp, D. 2009 Global mode interaction and pattern selection in the wake of a disk: a weakly nonlinear expansion. J. Fluid Mech. 633, 159-189.

Morgans, A. S. \& Dowling, A. P. 2007 Model-based control of combustion instabilities. J. Sound Vib. 299, 261-282.

Rigas, G., Morgans, A. S., Brackston, R. D. \& Morrison, J. F. 2015 Diffusive dynamics and stochastic models of turbulent axisymmetric wakes. J. Fluid Mech. 778, R2.

Rigas, G., Oxlade, A. R., Morgans, A. S. \& Morrison, J. F. 2014 Low-dimensional dynamics of a turbulent axisymmetric wake. J. Fluid Mech. 755, R5.

Risken, H. 1996 The Fokker-Planck Equation. Methods of Solutions and Applications, 2nd edn. Springer.

Skogestad, S. \& Postlethwaite, I. 2005 Multivariable Feedback Control. Analysis and Design, 2nd edn. John Wiley \& Sons Inc.

Volpe, R., Devinant, P. \& Kourta, A. 2015 Experimental characterization of the unsteady natural wake of the full-scale square back Ahmed body: flow bi-stability and spectral analysis. Exp. Fluids 56, 99.

ZwanzIG, R. 2001 Nonequilibrium Statistical Mechanics. Oxford University Press. 